\title{
The Influence of Planetary Magnetic Fields on Atmospheric Retention and Habitability
}

Primary Author

Dave Brain (University of Colorado; david.brain@colorado.edu; 303-735-5606)

Co-Authors

Rob Lillis (University of California Berkeley; rlillis@berkeley.edu, 510-508-7290)

Yingjuan Ma (University of California, Los Angeles; Yingjuan@ucla.edu; 310-825-5097)

Robin Ramstad (University of Colorado; robin.ramstad@colorado.edu; 303-735-7664)

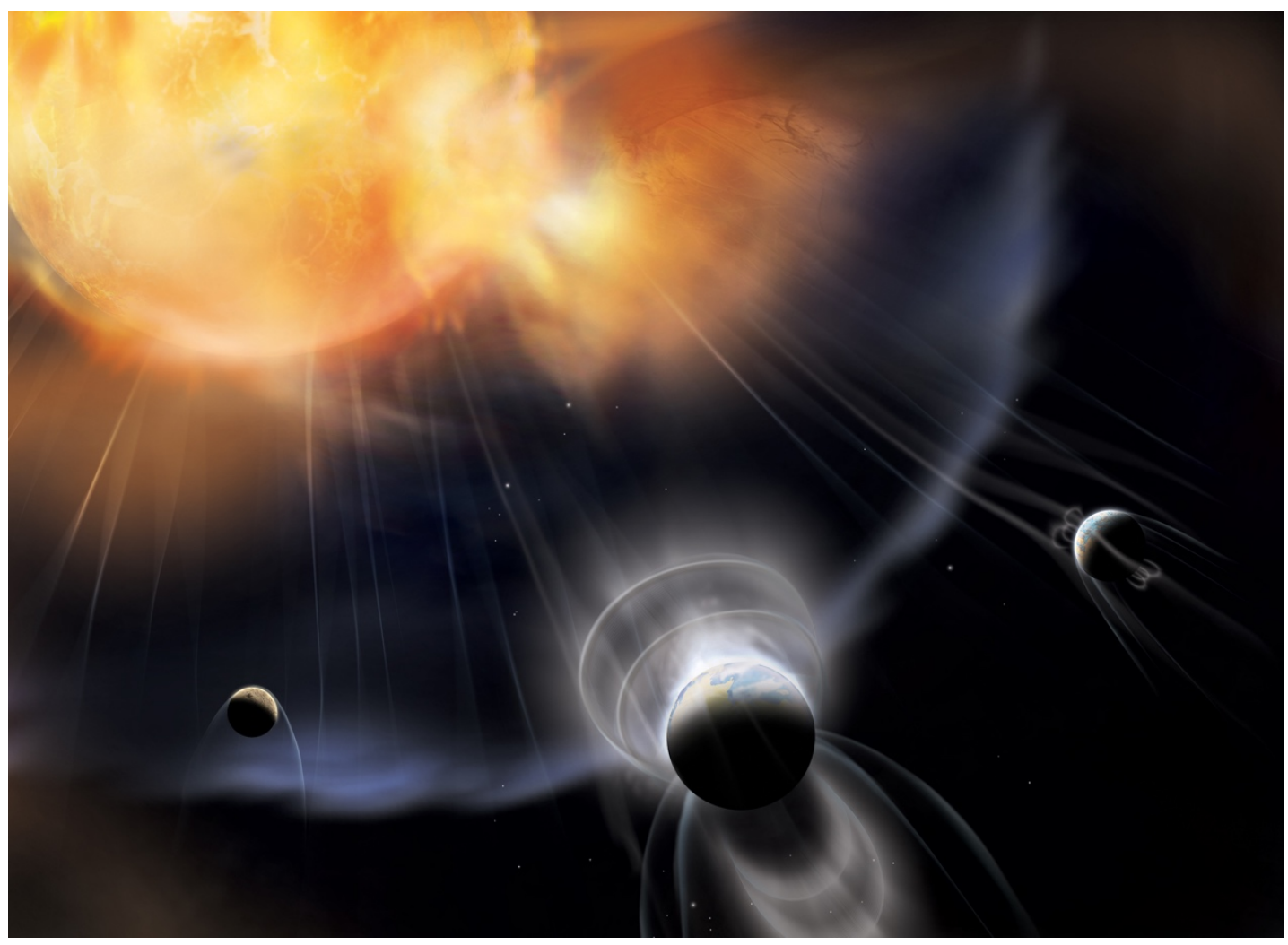




\section{Motivation}

The chain of logic connecting 'habitable world' to 'magnetic field' typically proceeds as follows:

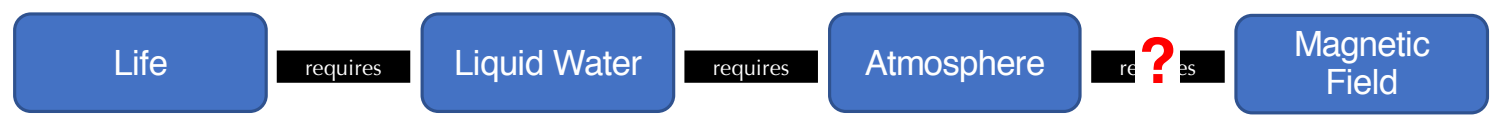

where each item to the right is a requirement for the item to its left. The connections are reasonable. All of the millions of species of life on Earth require liquid water. There must be sufficient pressure for water to exist as a liquid. And, until recently, it was widely accepted that a global magnetic field prevents an atmosphere from escaping to space.

However, each link in this chain also contains implicit assumptions. The first link assumes that all life is Earth-like, but other liquids such as ammonia or methane have been discussed as viable alternatives to water if life formed elsewhere [e.g. Benner et al., 2004]. The second link assumes that life only exists at a planet's surface, though subsurface life could (and on Earth, does) survive without any significant planetary atmosphere. The third link assumes that magnetic fields protect atmospheres from being stripped to space. This last assumption has been debated in recent years based on comparisons of in situ observations of atmospheric escape made at Venus, Earth, and Mars [e.g. Moore and Horwitz, 2007; Strangeway et al., 2010]. Planetary magnetic fields can influence habitability in other ways (discussed less in this white paper, but still relevant). For example, a strong planetary field should alter the fluxes of energetic charged particles into an atmosphere, perhaps changing its atmospheric chemistry [e.g. Airapetian et al., 2016]. A strong field can also shield a surface from highly energetic charged particle radiation that is harmful to life.

Understanding the extent to which planetary magnetic fields influence atmospheres and habitability will close knowledge gaps in several disciplines:

- Did the absence of persistent global magnetic fields on Mars and Venus contribute to their divergent climate histories relative to Earth? Is the significant historical atmospheric loss inferred for Mars [e.g. Jakosky et al., 2018] attributable primarily to its small size relative to Earth, to its lack of magnetic field, or both?

- Would atmospheric abundances for the solar system's large moons be different if the magnetization state of the moons were different?

- How does Earth's atmosphere behave during geomagnetic reversal periods?

- Which exoplanets are likely to retain significant atmospheres? It is still often assumed that a planet must have a magnetic field to retain a habitable atmosphere [e.g. Driscoll and Barnes, 2015]. Though exoplanetary plasma interactions are now being considered more fully [e.g. C. Dong et al., 2017a,b], the role of a magnetic field in exoplanet atmospheric retention and habitability is far from settled. For example, unmagnetized planets orbiting in the Habitable Zone of active M Dwarf stars are particularly exposed to stellar energetic inputs that could remove their atmospheres - would magnetized planets fare any better? 


\section{Current State of the Field}

In broad terms, any particle near the top of an atmosphere (where collisions are rare) can escape a planet's gravity if it is given enough energy and is directed "upward" [see reviews of atmospheric escape processes in, e.g., Moore and Horwitz, 2007; Brain et al., 2016]. For neutral particles this energy can come from heating by solar photons (only important today for hydrogen at Venus, Earth, and Mars), from chemical reactions (important today mostly for oxygen at Mars), or from collisions (not thought to be important at any of the three planets, at least today). However, since ions are charged particles, they have access to additional important sources of energy through interactions with electric fields and other charged particles. Ion escape can therefore be especially effective for heavier species that require substantial energy for escape. These species (e.g. $\mathrm{O}^{+}, \mathrm{O}_{2}{ }^{+}, \mathrm{N}_{2}{ }^{+}, \mathrm{CO}_{2}{ }^{+}$) are important for determining climate since they are (or are derived from) major atmospheric gases $\left(\mathrm{CO}_{2}, \mathrm{~N}_{2}\right)$. Magnetic fields influence the motion of charged particles, and therefore a strong planetary magnetic field will influence ion escape processes.

Early suggestions that global dipole fields protect an atmosphere from escaping to space were motivated by measurements from the early U.S. and Soviet missions to Mars and Venus that suggested that the two planets lacked significant global dipole fields [e.g. McElroy, 1968]. Over the ensuing decades there have been additional pieces of observational evidence that magnetic fields shield atmospheres from escaping. For example, the atmospheres of Venus and Mars are enriched in heavy isotopes such as deuterium - a signature of the removal of particles from the top of the atmosphere. This enrichment is greater than for Earth, suggesting that the two un-magnetized worlds have lost more atmosphere than magnetized Earth. In another example, observations of a single high solar wind pressure event encountering both Earth and Mars were argued to show that escape rates increased by only a small amount at magnetized Earth, but a large amount at unmagnetized Mars [Wei et al., 2012]. Most recently, observations from the Mars Atmosphere and Volatile EvolutioN (MAVEN) mission currently orbiting Mars (Figure 1) confirm the loss of substantial amounts of atmosphere over the history of the planet [Jakosky et al., 2018], consistent with the idea of efficient loss at an unmagnetized world.
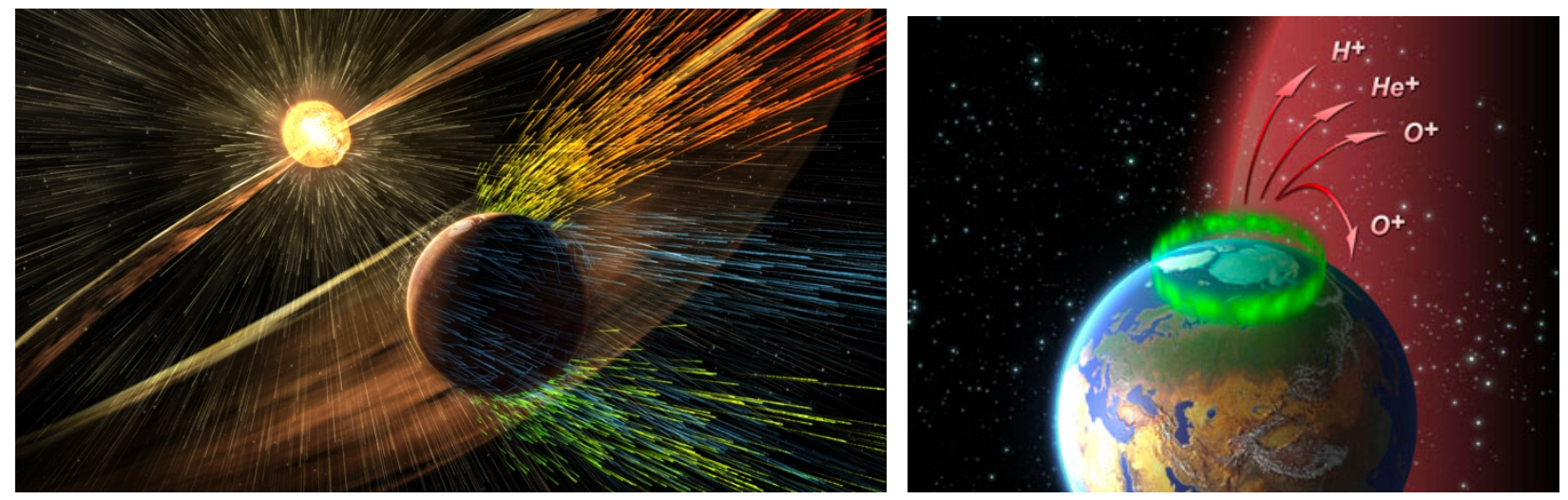

Figure 1: (left) Artist's sketch of atmospheric charged particles escaping from the Martian atmosphere in response to a Coronal Mass Ejection. MAVEN results confirm that atmospheric escape has played a major role in changing the Martian climate, possibly due to the lack of a global magnetic field at Mars. However, atmospheric ion escape occurs at Earth (right) and is correlated with the energy of the solar wind, despite - or perhaps enhanced by - the presence of Earth's global magnetic field. The escape is concentrated in the magnetic cusp regions. Image credits: NASA / MAVEN and ESA. 
Figure 2: Oxygen ion escape fluxes from the Earth [Nilsson et al., 2011] in a cylindrical coordinate system. Analogous maps of escaping $\mathrm{O}^{+}$flux from Venus [Fedorov et al., 2008] and Mars [Ramstad et al., 2017] are inlaid with the same scale. Escape flux estimates vary by study, but all lie within about an order of magnitude of each other, sparking debate about the importance of a magnetic field. The size of Earth's magnetosphere greatly alters the scale of the ion outflow. Calculating escape rates involves not only measuring flux, but also an estimate of the crosssectional area of the outflow.

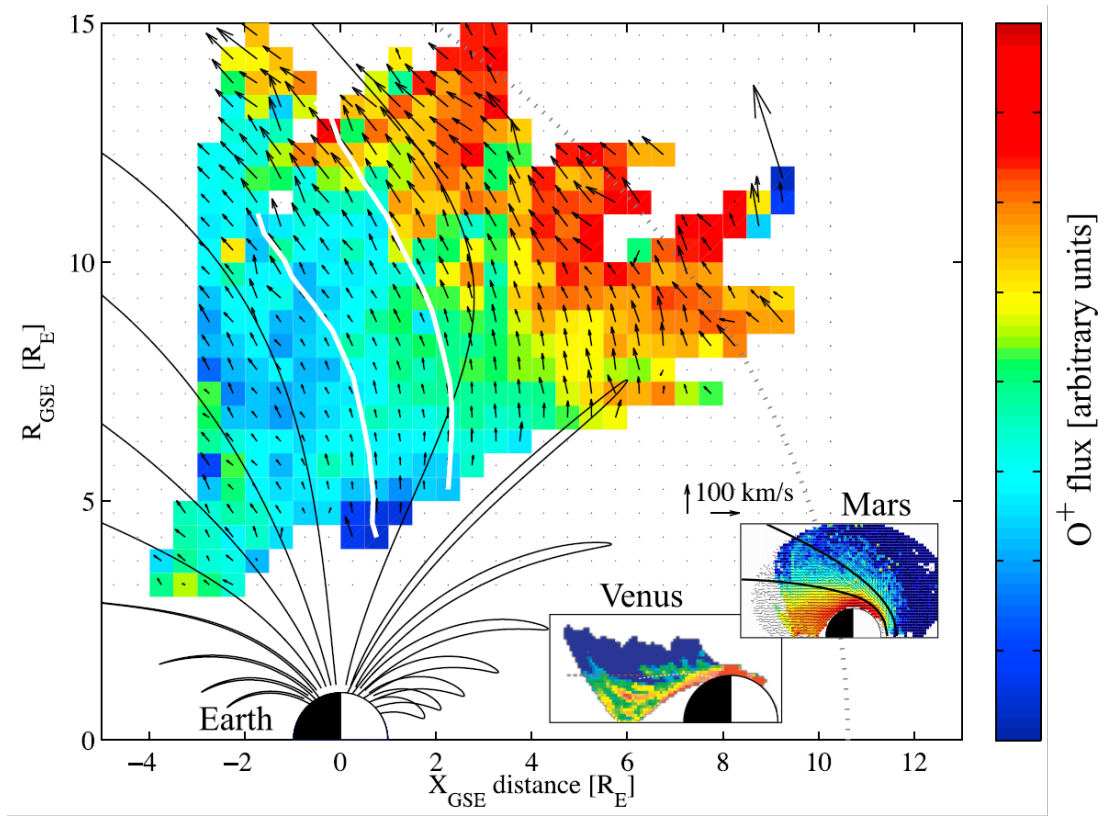

The idea that planetary scale magnetic fields lead to reduced escape rates has been recently questioned. One of the first to propose that magnetic fields do not protect atmospheres was Strangeway et al. [2010], who noted that the reported ion escape rates at Venus, Earth, and Mars agree to within about an order of magnitude (Figure 2). This suggests that atmospheric escape is not far more efficient at unmagnetized worlds. It has also been shown that ion outflow in Earth's magnetic cusps is proportional to the Poynting flux in the solar wind [Strangeway et al., 2005; Li et al., 2017], suggesting a direct link between the solar wind energy and the energy of escaping ions (Figure 1).

Strangeway et al. [2010] argued that an intrinsic dipole field encounters more power from the solar wind than an unmagnetized planet due to the large size of the magnetosphere relative to the planet, and that solar wind energy will be transferred along magnetospheric field lines to the top of an atmosphere in the cusp regions, possibly resulting in more ion escape than results in the absence of a global field. But the basic premise that magnetic fields prevent the solar wind from accessing upper atmospheres is also difficult to dismiss.

Initial modeling investigations suggest that neither argument is fully correct. Atmospheric ion escape is not purely correlated or anti-correlated with increasing planetary magnetic field strength. Instead, these global plasma models hint that atmospheric escape rates are maximized for intermediate magnetic field strengths [e.g. Egan et al., 2019; Sakata et al., 2020]. Too strong a magnetic field prevents outflowing planetary ions from escaping the system, while too weak a magnetic field results in planetary ions being accelerated by the solar wind back into the atmosphere. However, these models do not capture all of the relevant physics, and are often limited by computational resources to relatively weak planetary magnetic field strengths. 


\section{Needs}

How might we resolve the question of magnetic field influence on planetary atmospheres and habitability? We can appeal to observations, simulations, or theory. Each approach has been pursued in recent years with interesting but contradictory results [e.g. Wei et al., 2012; Sakai et al., 2018; Gunnell et al., 2018]. More work is needed in each area. The main needs are listed below, without regard to priority - an ideal outcome would be comparable progress in all four identified areas.

\section{A. Directly comparable measurements of atmospheric energization and escape at a variety of magnetized and unmagnetized solar system objects, along with simultaneous measurements of the solar inputs that drive escape}

Measurement-based estimates of atmospheric escape have been made for many solar system objects (e.g. Venus, Earth, Mars, Titan, comets, Pluto). Several caveats make directly comparing published escape rates between planets problematic. First, published escape rates vary depending upon the instrument capabilities, spacecraft orbit, data selection, and data treatment. Second, there is also natural variability in escape rates with different drivers associated with the solar Extreme Ultraviolet (EUV) flux [e.g. Kollmann et al., 2016; Ramstad et al., 2015], and solar wind [e.g. Slapak et al., 2017; Ramstad et al., 2017; Persson et al., 2020]. This suggests that the time period over which data were taken influences the escape rate. Third, the range of solar and solar wind drivers experienced by these objects is different, even over the same period, due to their different heliocentric distances and longitudes (Figure 3).

The most comprehensive set of measurements to date has been made by the MAVEN spacecraft at Mars. MAVEN has been measuring escape rates via the full suite of escape processes active at Mars, and simultaneously measuring the solar and solar wind energetic inputs that drive escape (EUV and X-ray photon fluxes, solar wind and solar energetic particle energy fluxes, interplanetary magnetic field and associated magnetic pressure). There is, at present, no analogous dataset for other solar system bodies of interest, including Venus, Earth, Titan, comets, Pluto, or giant planets.

Comparing atmospheric escape from a variety of objects with a range of sizes (from comet to giant planet), external conditions, and magnetization states is likely the best way to make forward progress in determining the importance of a magnetic field. For this comparison to be meaningful we must understand (and if possible control or correct for) differences in measurement technique, instrument performance, solar inputs, and planetary characteristics. A particularly helpful situation would be simultaneous measurements of escape made at multiple solar system objects by similar instruments.

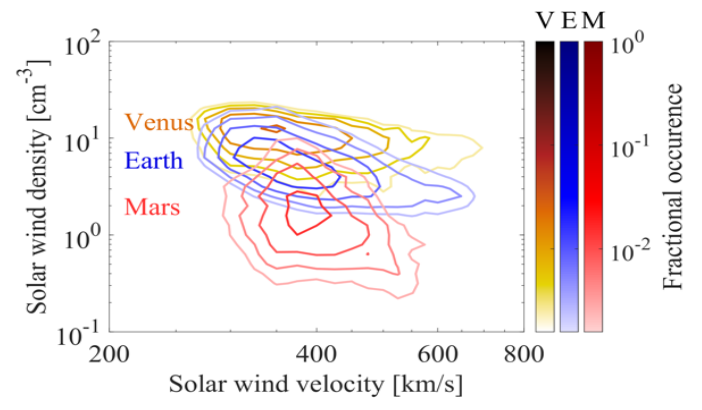

Figure 3: Distributions of solar wind parameters upstream at Venus (yellow, Venus Express), Earth (blue, ACEMIND), and Mars (red, Mars Express) showing that ion escape at the three planets occurs under different solar wind driving conditions 


\section{B. Development and validation of models that bridge the gap between magnetized and unmagnetized planets}

Physics-based global magnetospheric models provide a powerful means of computing ion escape rates from planets. Unlike spacecraft data, models tell us about the entire system at any given moment, and can be used to probe the physics responsible for energizing and removing ions. Models can vary a single parameter (e.g. the intensity of the planetary dipole moment) to determine its influence on the system. We require a model that includes proper physical processes and is validated by observations. This model should be able to handle both unmagnetized and magnetized planets with varying dipole field strength. To date, such a model does not exist.

Magnetized and unmagnetized planets are currently typically treated with different models, and the two scientific communities are largely separate. For unmagnetized planets, where the solar wind interacts with the ionosphere directly, an ionosphere needs to be included self-consistently in order to properly simulate the interaction process and reproduce observed features [e.g. Ma et al., 2013]. For magnetized planets, modeling ion outflow is an extremely challenging task. There is a gap in our understanding of the source and energization mechanisms in the ionosphere, and transport to the magnetosphere, largely as a result of the complex interplay between the solar wind, magnetospheric activity, and the ionosphere. Glocer et al. [2018] only recently presented the first two-way coupled ionospheric outflow-magnetosphere model for Earth including kinetic ion effects.

One of the major reasons that the Earth and unmagnetized planetary modeling communities have diverged is the difficulty in simulating plasma motion in strong magnetic fields (Alfvén speeds are high in strong magnetic field, requiring a small timestep that makes long-term calculation difficult) [Toth et al., 2012]. Steady advances in computational resources over the past decade or so have made it possible for the more self-consistent models for unmagnetized planets to add weak planetary magnetic fields. It has recently become possible for Venus-like MHD models to simulate dipole fields $\sim 30 \%$ of Earth-strength - but the models are not validated in this regime and there may be missing or poorly-captured physics in the low-altitude cusp regions most important for ion acceleration and escape.
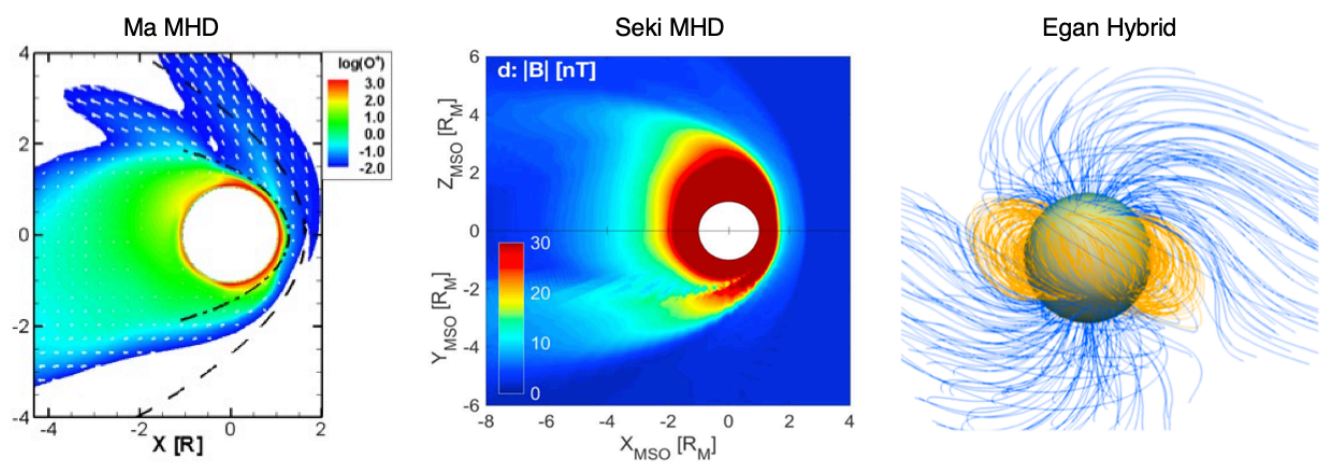

Figure 4: Example simulation results from three global plasma models. All simulations shown are for Mars-like planets. The Seki and Egan simulations show results with a weak global dipole magnetic field included. 


\section{A theoretical framework that unifies the current understanding at both magnetized and unmagnetized planets}

If we wish to apply our understanding of magnetic field influences to early solar system or exoplanetary situations (where we have no measurements of ion escape and for which models are not validated), then we require a conceptual framework for ion loss that applies to a planet with any intrinsic magnetic field strength (including none at all). This framework would be able to track the flow of energy and ions throughout a planet's ionosphere and magnetosphere for both magnetized and unmagnetized planets. It would include all relevant energy sources, and all relevant pathways and processes for ion escape. Examples approaching this kind of framework were presented for Earth and magnetized planets by Strangeway et al., [2005] and Moore and Horwitz, [2007] (Figure 5), but they are overly simplified. A general framework that unifies our understanding of escape from magnetized and unmagnetized planets has not been developed.
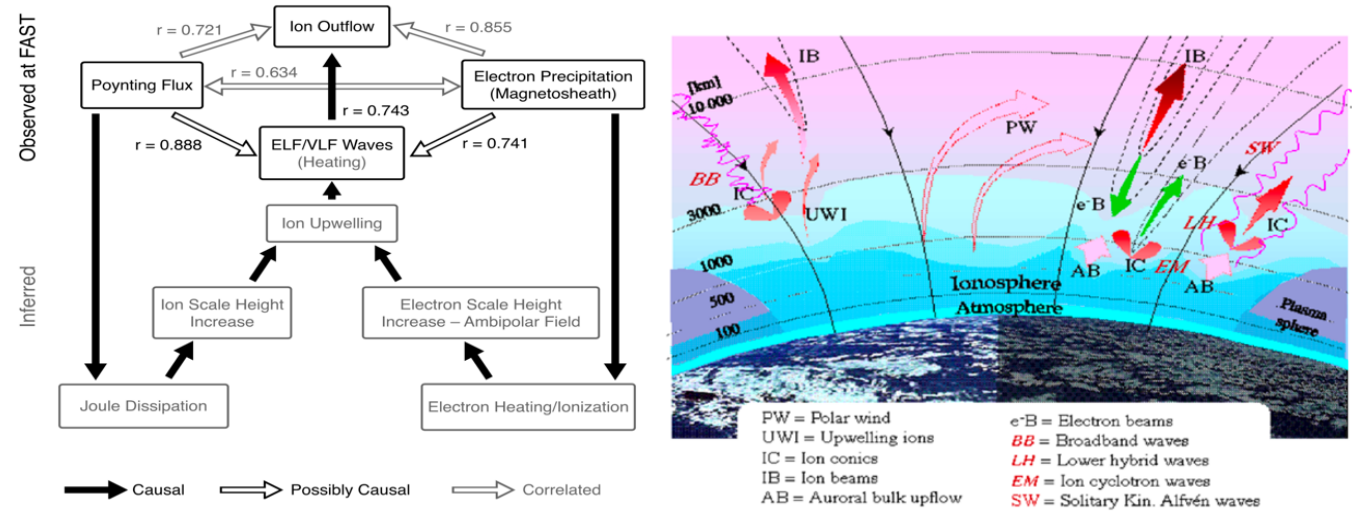

Figure 5: Examples of schematic conceptual frameworks for ion loss showing (left) the flow of energy and (right) processes relevant for ion outflow in Earth's ionosphere and magnetosphere. From Strangeway et al., [2005] and Moore and Horwitz, [2007]. These diagrams are simplified.

\section{An interdisciplinary approach that enables contributions from Planetary Science, Heliophysics, Astrophysics, and Earth Science}

The inclusion of measurements from multiple planets, detailed simulations, and conceptual theoretical models is essential for the success of such an approach. A second essential element is the inclusion of experts from both the unmagnetized and magnetized planetary atmospheric escape communities, as well as from the exoplanetary atmospheric escape community. These three communities have diverged over the past several decades, though they study many of the same processes in different settings. When the influence of magnetic fields on atmospheric chemistry is included, a fourth community (Earth Science) must be folded in as well. By promoting discussion and collaboration between the communities, we will be able to better answer our core question. By collaborating on an answer, we will be better positioned to apply it within our respective communities. NASA's NExSS community provides one example of cross-divisional collaboration, albeit with an exoplanet focus. These kinds of efforts should be expanded, or adapted for the specific needs of Planetary Science. 


\section{References}

Airapetian, V.S., et al. (2019), "Impact of Space Weather on Climate and Habitability of Terrestrial Type Exoplanets." International Journal of Astrobiology.

Benner, S. A., A. Ricardo and M. A. Carrigan (2004). "Is there a common chemical model for life in the universe?" Current Opinion in Chemical Biology 8(6): 672-689.

Brain, D. A., et al. (2016). "Atmospheric escape from unmagnetized bodies." Journal of Geophysical Research-Planets 121(12): 2364-2385.

Dong, C. F., et al. (2017). "Is Proxima Centauri b Habitable? A Study of Atmospheric Loss." Astrophysical Journal Letters 837(2).

Driscoll, P. E. and R. Barnes (2015). "Tidal Heating of Earth-like Exoplanets around M Stars: Thermal, Magnetic, and Orbital Evolutions." Astrobiology 15(9): 739-760.

Egan, H. et al. (2019), "Planetary Magnetic Field Control of Ion Escape from Weakly Magnetized Planets", Monthly Notices of the Royal Astronomical Society.

Fedorov, A., et al. (2008). "Comparative analysis of Venus and Mars magnetotails." Planetary and Space Science 56(6): 812-817.

Glocer, A., G. Toth and M. C. Fok (2018). "Including Kinetic lon Effects in the Coupled Global Ionospheric Outflow Solution." Journal of Geophysical Research-Space Physics 123(4): 2851-2871.

Gunell, H., et al. (2018). "Why an intrinsic magnetic field does not protect a planet against atmospheric escape." Astronomy \& Astrophysics 614.

Jakosky, B. M., D. Brain, et al. (2018). "Loss of the Martian atmosphere to space: Present-day loss rates determined from MAVEN observations and integrated loss through time." Icarus 315: 146-157.

Kollmann, P., et al. (2016). "Properties of planetward ion flows in Venus' magnetotail." Icarus 274: 73-82.

Lammer, H., et al. (2008). "Atmospheric Escape and Evolution of Terrestrial Planets and Satellites." Space Science Reviews 139(1-4): 399-436.

$\mathrm{Li}$, K., et al. (2017). "Cold Ion Outflow Modulated by the Solar Wind Energy Input and Tilt of the Geomagnetic Dipole." Journal of Geophysical Research-Space Physics 122(10): 10658-10668.

Ma, Y. J., et al. (2013). "A global multispecies single-fluid MHD study of the plasma interaction around Venus." Journal of Geophysical Research-Space Physics 118(1): 321-330.

Mcelroy, M. B. (1968). "Upper Atmosphere of Venus." Journal of Geophysical Research 73(5): 1513-\&.

Moore, T. E. and J. L. Horwitz (2007). "Stellar ablation of planetary atmospheres." Reviews of Geophysics 45(2).

Nilsson, H. (2011). "Heavy Ion Energization, Transport, and Loss in the Earth's Magnetosphere." Dynamic Magnetosphere 3: 315-327.

Persson, M., et al. (2020). "The Venusian atmospheric oxygen ion escape: Extrapolation to the early Solar System, Journal of Geophysical Research-Planets 125(3): e2019JE006336.

Ramstad, R., et al. (2017). "Global Mars-solar wind coupling and ion escape." Journal of Geophysical Research-Space Physics 122(8): 8051-8062.

Ramstad, R., et al. (2015). "The Martian atmospheric ion escape rate dependence on solar wind and solar EUV conditions: 1. Seven years of Mars Express observations." Journal of Geophysical Research-Planets 120(7): 1298-1309.

Sakai, S., et al. (2018). "Effects of a Weak Intrinsic Magnetic Field on Atmospheric Escape From Mars." Geophysical Research Letters 45(18): 9336-9343.

Strangeway, R.S., et al. (2010), "Does a Planetary-Scale Magnetic Field Enhance or Inhibit lonospheric Plasma Outflows?", AGU Fall Meeting Abstracts, SM33B-S1893.

Strangeway, R. J., et al. (2005). "Factors controlling ionospheric outflows as observed at intermediate altitudes." Journal of Geophysical Research-Space Physics 110(A3).

Tóth, G., et al. (2012). Adaptive numerical algorithms in space weather modeling. Journal of Computational Physics, 231(3), 870-903. https://doi.org/10.1016/j.jcp.2011.02.006

Wei, Y., et al. (2012). "Enhanced atmospheric oxygen outflow on Earth and Mars driven by a corotating interaction region." Journal of Geophysical Research-Space Physics 117. 